\title{
CONTINUOUS-TIME SKEWED MULTIFRACTAL PROCESSES AS A MODEL FOR FINANCIAL RETURNS
}

\author{
EMMANUEL BACRY, ${ }^{*}$ CNRS and École Polytechnique \\ LAURENT DUVERNET, ${ }^{* *}$ Université Paris-Est Marne-la-Vallée and École Polytechnique \\ JEAN-FRANÇOIS MUZY, ${ }^{* * *}$ CNRS, Université de Corse, and École Polytechnique
}

\begin{abstract}
We present the construction of a continuous-time stochastic process which has moments that satisfy an exact scaling relation, including odd-order moments. It is based on a natural extension of the multifractal random walk construction described in Bacry and Muzy (2003). This allows us to propose a continuous-time model for the price of a financial asset that reflects most major stylized facts observed on real data, including asymmetry and multifractal scaling.
\end{abstract}

Keywords: Multifractal process; skewness; leverage effect

2010 Mathematics Subject Classification: Primary 91B25; 91B24; 60G18

\section{Introduction}

A striking feature of the prices of financial assets is that a certain number of statistical properties, generally referred to as 'stylized facts', appear to be universal. Indeed, it is for instance well known that, for any considered asset and any time scale from a few minutes to a few months or years, the log-returns are a centered and uncorrelated time series with a heavytailed distribution, and that the absolute or squared log-return time series presents long memory and persistence. We refer the reader to [5] and [10] for a thorough review of the various statistical properties that can be observed on financial data. Formulating a probabilistic, continuous-time model that reflects most of these stylized facts is naturally of first importance, both from a theoretical and practical point of view, and has been the motivation of a considerable number of research papers.

Many recent empirical studies have also suggested that financial data share statistical properties with turbulent intermittent velocity fields [3], [5], [11]: areas of rapid and violent activities alternate with more peaceful ones, and this phenomenon repeats itself at any time scale in the 'same' way. So as to take this elaborate scale invariance into account, a natural approach is then to suppose that the local Hölder regularity of the underlying continuous signal is itself random, which also translates into a 'multiscaling' or a 'multifractal scaling' of price return fluctuations;

Received 19 July 2011; revision received 16 December 2011.

* Postal address: Centre de Mathématiques Appliquées UMR 7641, École Polytechnique CNRS, Route de Saclay, 91128 Palaiseau Cedex, France. Email address: emmanuel.bacry@ polytechnique.fr

** Postal address: Laboratoire MODAL'X, Département de Mathématiques, UFR SEGMI, Université Paris OuestNanterre, 200 avenue de la République, 92001 Nanterre Cedex, France.

Email address: duvernet@cmap.polytechnique.fr

*** Postal address: Laboratoire SPE UMR 6134, Université de Corse CNRS, 20250 Corte, France.

Email address: muzy@univ-corse.fr 
see [12] and[13]. A process $X$ with stationary increments is said to have a multifractal scaling if it satisfies

$$
\mathrm{E}\left[|X(t)|^{q}\right] \sim c_{q} t^{\zeta q} \quad \text { as } t \rightarrow 0
$$

for all $q$ s in some real interval, some positive constants $c_{q}$, and a scaling exponent $q \mapsto \zeta_{q}$ that is nonlinear. Since the pioneering work of Mandelbrot [15], the phenomenology of such multifractal models has provided new concepts and tools to analyze market fluctuations. It notably inspired the family of so-called 'cascade' random processes that account for the main statistical properties of financial prices in an elegant and parsimonious way [7], [17] (see also [4] for a nonfinancial approach). Moreover, these models are amenable to many analytical computations: they are easy to estimate and they lead to simple and yet very competitive solutions to the problem of conditional risk (volatility or value-at-risk) forecasting (see [3], [7], and [11]). However, although these multifractal models reproduce all the stylized facts we have already mentioned, we would like to point out one stylized fact that is not taken into account by them or indeed by most mathematical models, namely the leverage effect.

The so-called leverage effect is a feature that is mostly associated with stock and index prices: the variation of the log-return in the past is found to be negatively correlated to the volatility in the future (see [6]). Here, the volatility may be defined, for instance, as the squared or absolute log-return. So, basically, this effect quantifies the 'panic' effect that takes place after a large downward move of the price which tends to increase the volatility much more than a large upward move would. Let us note that this effect induces two types of asymmetry in the price process. The first type (time asymmetry) is such that the price process is not invariant under reversion of time. Indeed, the variation of the volatility in the past is not correlated to the variation of the log-return in the future (if it were, then a simple arbitrage could actually be performed). The second type (return asymmetry) is such that this effect implies a negative skewness of the distribution of log-returns. The higher the leverage effect, the higher the skewness, the higher the asymmetry of the implied volatility smile (see [5]).

It therefore clearly appears important to incorporate these asymmetries into a probabilistic model of log-returns. Let us note that it has already been done in a nonmultifractal setting in, for instance, [6], [9], [16], and [18]. However, explicitly constructing a skewed multifractal process with leverage effect has not yet been done, though we should mention two very interesting works. In [19] the authors built a skewed model with some multiscaling properties and leverage effect in discrete time. However, when the sampling pace goes to 0 , even though the multiscaling properties converge to standard multifractal behavior (1), the skewness tends to 0 so that any leverage effect unfortunately disappears. The study in [20] was conducted in the setting of a turbulence study and not of finance, and as such the authors were not interested in this leverage effect.

In the present paper we try to fill this gap, and show how one can obtain a skewed, continuoustime, multifractal model for log-returns that reproduces all stylized facts mentioned in this introduction, including the leverage effect. Moreover, this modelization may be described as 'parsimonious' insofar as it relies only on a very small number of scalar parameters. So as to briefly summarize our approach, let us say that we extend the so-called multifractal random walk (MRW) model, which is one of the simplest multifractal, continuous 'cascade' models [3], [17], by introducing some explicit correlations between log-returns and volatility. However, by doing so, we also introduce correlations between log-returns, which is an undesirable feature when modeling the price of a financial asset. As we argue below, one can find a regime for the parameters of our model where these correlations are almost 0 , while the leverage effect remains quite noticeable. Hence, the statistical properties of real financial data match rather 
closely those of our model, as we are able to check using simulations. It should however be noted that obtaining a continuous-time multifractal random model with leverage effect and uncorrelated increments remains an open problem, and we hope that the present study is a helpful step toward solving it.

The paper is organized as follows. In Section 2 we present a brief overview of the (symmetrical) multifractal log-normal MRW model for financial data and discuss the first attempt to input asymmetry in this model [19]. In Section 3 we propose a new construction of a continuoustime, skewed, multifractal process that depends on a Hurst exponent $H>\frac{1}{2}$. In Section 4 we investigate its scaling properties and the behavior of all $q$-order moments (including the moments of order three and the skewness). In Section 5 we explicitly compute the leverage effect and discuss the choice of the parameter $H$, which affects both the correlation of the increments (which should be close to 0 , as in the case of financial data), and the skewness and the leverage effect (which should be significantly nonzero). A simulation scheme and some numerical simulations are presented in Section 6, which also contains a comparison with real data. Some computations and proofs that are used in the rest of the paper are postponed to Appendices A-C.

\section{Multifractal processes}

Let $X=(X(t), t \geq 0)$ be a real-valued stochastic process with stationary increments. For $t \geq 0$ and $\tau>0$, we write $\delta_{\tau} X(t)$ for the increment $X(t+\tau)-X(t)$. When the moments of order $p$ of $X$ satisfy

$$
\mathrm{E}\left[\left|\delta_{\tau} X(t)\right|^{q}\right] \approx c_{q} \tau^{\zeta q}
$$

for (small) $\tau>0$, it is usual to speak of either a monofractal scaling if the exponent $\zeta_{q}$ is a linear function of $q$, or a multifractal scaling if it is nonlinear.

\subsection{The MRW}

Muzy et al. [17] proposed the construction of a continuous-time stochastic random process that exhibits features quite similar to most stylized facts observed on the returns of financial assets (see [3]), including multifractal scaling, but excluding the leverage effect. The MRW $X(t)$ can be defined as the continuous limit as $n$ goes to $+\infty$ of the discretized process

$$
X_{n}(t)=\sum_{k=0}^{\lfloor n t\rfloor} \varepsilon_{n}\left(\frac{k}{n}\right) \mathrm{e}^{\omega_{n}(k / n)},
$$

where the $\varepsilon_{n}(k / n) \mathrm{s}$ are independent Gaussian random variables with mean equal to 0 and variance equal to $\sigma^{2} / n$ for some $\sigma>0$, and $\omega_{n}$ is a Gaussian stationary process independent of the $\varepsilon_{n}(k) \mathrm{s}$. Alternatively, we can consider the continuous construction

$$
X(t)=\sigma \lim \int_{0}^{t} \mathrm{e}^{\omega_{n}(u)} \mathrm{d} B(u) \quad \text { as } n \rightarrow+\infty,
$$

where $B$ is a standard Brownian motion independent of $\omega_{n}$; see [2]. The autocovariance of $\omega_{n}$ is given by

$$
\operatorname{cov}\left[\omega_{n}\left(\frac{j}{n}\right), \omega_{n}\left(\frac{k}{n}\right)\right]= \begin{cases}\lambda^{2} \log \frac{n T}{|j-k|+1} & \text { if } \frac{|j-k|+1}{n} \leq T, \\ 0 & \text { otherwise, }\end{cases}
$$

and the expectation of $\omega_{n}$ is such that $\mathrm{E}\left[\mathrm{e}^{2 \omega_{n}(\cdot)}\right]=1$. Here $\lambda^{2}>0$ is a parameter called the 
intermittency coefficient, and $T>0$ is a parameter (called the integral scale) such that scaling (2) holds exactly for all $\tau \in[0, T]$. More precisely, as shown in [2], the following relation holds for all $r \in[0,1]$ :

$$
(X(r t), 0 \leq t \leq T) \stackrel{\text { law }}{=} r^{1 / 2} \mathrm{e}^{\Omega_{r}}(X(t), 0 \leq t \leq T) .
$$

Here $\Omega_{r}$ is a Gaussian random variable independent of $X$, with expectation $-\lambda^{2} \log \left(r^{-1}\right)$ and variance $\lambda^{2} \log \left(r^{-1}\right)$. We can then deduce that the multifractal scaling

$$
\mathrm{E}\left[\left|\delta_{\tau} X(t)\right|^{q}\right]=c_{q} \tau^{\zeta q}
$$

is satisfied for $\tau \in[0, T]$, with $\zeta_{q}=q / 2-\lambda^{2}\left(q-q^{2} / 2\right)$ and $c_{q}=\mathrm{E}\left[|X(T)|^{q}\right] T^{-\zeta_{q}}$ for all $q$ s such that $c_{q}$ is finite. It was furthermore shown in [2] that $c_{q}$ is finite for all $q \in\left(0, \lambda^{-2}\right)$ and $c_{q}=+\infty$ for all $q>\lambda^{-2}$, so the process $X$ does not have moments of all orders and the distribution of $X(t)$ is heavy tailed.

\subsection{Further extensions: towards a skewed model with leverage effect}

The MRW model has been shown to have interesting applications to financial data, in particular in terms of volatility and value-at-risk forecasting; see [3], [7], and [11]. However, one significant drawback of the MRW approach is that the distribution of $X(t)$ is symmetric, so it does not reflect the skewness empirically observed on financial data. Moreover, since the two processes $\varepsilon$ and $\omega$ are independent, it follows that the increments of the process $X$ and the square of the increments are uncorrelated. Therefore, the model does not present the 'leverage effect' observed in stocks and financial index prices.

Pochart and Bouchard [19] therefore proposed modifying the construction (3) as

$$
\tilde{X}_{n}(t)=\sum_{k=0}^{\lfloor n t\rfloor} \varepsilon_{n}\left(\frac{k}{n}\right) \exp \left(\omega_{n}\left(\frac{k}{n}\right)-n^{\alpha} \sum_{i<k} K\left(\frac{i}{n}, \frac{k}{n}\right) \varepsilon\left(\frac{i}{n}\right)\right),
$$

where $K$ is a positive kernel. As they showed, this leads to a fairly well-reproduced leverage effect observed on the data, as well as retaining most of the nice properties of the original MRW. However, the authors noted that this holds only for $n<+\infty$ : indeed, the odd moments of the process vanish when $n$ goes to $+\infty$, so their approach only gives a discrete-time model for $t=0,1 / n, \ldots, i / n, \ldots$. Another related work is that of Robert and Vargas [20], who studied a skewed three-dimensional generalization of the MRW model with applications to hydrodynamics.

In what follows we propose an alternate, continuous-time construction for a multifractal random walk with skewness and leverage effect. In particular, we modify construction (3) by defining the noise $\varepsilon$ as a fractional Gaussian noise with Hurst exponent $H$, where $H$ is chosen in a regime where the increments of $X$ are almost uncorrelated. Thus, our approach shares some connections with the previous works of Abry et al. [1] and Ludeña [14], who also considered the question of constructing an MRW with fractional noise. However, in these papers the noise $\varepsilon$ was independent of the volatility process $\omega$, whereas we will define them as correlated processes.

\section{Construction of a continuous-time skewed MRW}

\subsection{Definition of the skewed process}

Fix the following parameters: $\lambda \in\left(0, \frac{1}{2}\right), T>0, \sigma>0$, and $H \in\left(\frac{1}{2}+\lambda^{2} / 2,1\right)$. The parameters $\lambda, T$, and $\sigma$ are of a similar nature as above, while $H$ can be seen as a Hurst 
exponent, as in [1] and [14]. We define a skewed multifractal random walk by

$$
X(t)=\lim X_{l}(t) \quad \text { as } l \rightarrow 0,
$$

where

$$
X_{l}(t)=\int_{0}^{t} \varepsilon_{l}(u) \mathrm{e}^{\omega_{l}(u)} \mathrm{d} u
$$

and $(\varepsilon, \omega)=\left(\left(\varepsilon_{l}(u), \omega_{l}(u)\right), u \in \mathbb{R}, l \in(0, T)\right)$ is a Gaussian process with values in $\mathbb{R}^{2}$ that satisfies the following properties.

Property 1. $\left(\varepsilon_{l}(u), \omega_{l}(u)\right)$ is stationary in $u$, that is, for $u_{1}, \ldots, u_{n}, \tau \in \mathbb{R}$,

$$
\left(\left(\varepsilon_{l}\left(u_{1}\right), \omega_{l}\left(u_{1}\right)\right), \ldots,\left(\varepsilon_{l}\left(u_{n}\right), \omega_{l}\left(u_{n}\right)\right), l \in(0, T)\right)
$$

and

$$
\left(\left(\varepsilon_{l}\left(u_{1}+\tau\right), \omega_{l}\left(u_{1}+\tau\right)\right), \ldots,\left(\varepsilon_{l}\left(u_{n}+\tau\right), \omega_{l}\left(u_{n}+\tau\right)\right), l \in(0, T)\right)
$$

have the same law.

Property 2. $\left(\varepsilon_{l}(u), \omega_{l}(u)\right)$ has independent increments in $l$, that is, for $l^{\prime}<l$,

$$
\left(\left(\varepsilon_{l^{\prime}}(u)-\varepsilon_{l}(u), \omega_{l^{\prime}}(u)-\omega_{l}(u)\right), u \in \mathbb{R}\right) \quad \text { and } \quad\left(\left(\varepsilon_{l}(u), \omega_{l}(u)\right), u \in \mathbb{R}\right)
$$

are independent.

Property 3. The expectation of $\omega_{l}(u)$ is $-\frac{1}{2} \operatorname{var}\left[\omega_{l}(u)\right]\left(\right.$ so $\left.\mathrm{E}\left[\mathrm{e}^{\omega_{l}(u)}\right]=1\right)$. The expectation of $\varepsilon_{l}(u)$ is 0 .

Note that the normalization of $\omega$ is thus different from the MRW case presented in the previous section.

Property 4. For $\tau \in \mathbb{R}$, let us write $\gamma_{l}^{\omega}(\tau), \gamma_{l}^{\varepsilon}(\tau)$, and $\gamma_{l}^{\omega \varepsilon}(\tau)$ respectively for

$$
\operatorname{cov}\left[\omega_{l}(u), \omega_{l}(u+\tau)\right], \quad \operatorname{cov}\left[\varepsilon_{l}(u), \varepsilon_{l}(u+\tau)\right], \quad \text { and } \quad \operatorname{cov}\left[\varepsilon_{l}(u), \omega_{l}(u+\tau)\right],
$$

and let us define

$$
\begin{gathered}
\gamma^{\omega}(\tau)=\lambda^{2} \max \left(\log \left(\frac{T}{|\tau|}\right), 0\right), \\
\gamma^{\varepsilon}(\tau)=c^{\varepsilon} \sigma^{2}|\tau|^{-2+2 H}, \\
\gamma^{\omega \varepsilon}(\tau)=c^{\omega \varepsilon} \sigma \lambda(\max (\tau, 0))^{-1+H},
\end{gathered}
$$

for some positive constants $c^{\varepsilon}$ and $c^{\omega \varepsilon}$ (we use the convention that $0^{-1}=+\infty$ ). Then, for fixed $\tau, \gamma_{l}^{\omega}(\tau) \uparrow \gamma^{\omega}(\tau), \gamma_{l}^{\varepsilon}(\tau) \uparrow \gamma^{\varepsilon}(\tau)$, and $\gamma_{l}^{\omega \varepsilon}(\tau) \uparrow \gamma^{\omega \varepsilon}(\tau)$ as $l$ goes to 0 . Moreover, $\gamma_{l}^{\omega \varepsilon}(\tau)$ is 0 for $l \in(0, T)$ and $\tau \leq 0$.

Property 5. We have the following scaling equations for $l \in(0, T), \tau \in[-T, T]$, and $r \in$ $(0,1]$ :

$$
\begin{gathered}
\gamma_{r l}^{\omega}(r \tau)=-\lambda^{2} \log (r)+\gamma_{l}^{\omega}(\tau), \\
\gamma_{r l}^{\varepsilon}(r \tau)=r^{-2+2 H} \gamma_{l}^{\varepsilon}(\tau),
\end{gathered}
$$

and

$$
\gamma_{r l}^{\omega \varepsilon}(r \tau)=r^{-1+H} \gamma_{l}^{\omega \varepsilon}(\tau)
$$


As explained below, these properties are sufficient to prove the convergence $X_{l} \rightarrow X$ and to study the properties of $X$. However, we still need to justify the existence of such a process $(\varepsilon, \omega)$; this is done in Subsection 3.3 where we explicitly construct an example of $(\varepsilon, \omega)$ that satisfies the above properties. The exact values of $\gamma_{l}^{\omega}(\tau), \gamma_{l}^{\varepsilon}(\tau)$, and $\gamma_{l}^{\omega \varepsilon}(\tau)$ for $\tau \in \mathbb{R}$ and $l \in(0, T)$, as well as the constants $c^{\varepsilon}$ and $c^{\omega \varepsilon}$ corresponding to this construction can be found in Appendix A.

Remark. It can be immediately seen that the function $\gamma^{\varepsilon}$ in Property 4 is the covariance of a fractional Brownian noise with Hurst exponent $H$, so the process $\left(\int_{0}^{t} \varepsilon_{l}(u) \mathrm{d} u, t \geq 0\right)$ converges in law to a fractional Brownian motion as $l$ goes to 0 . (We could easily prove that the convergence also holds under stronger modes; however, this is of little interest for our purpose here.)

\subsection{Existence of $X$}

In this subsection we prove the existence and nondegeneracy of the process $X$. Let us define the following condition on $p \geq 2$ :

$(\mathscr{H}(p)) p H-\lambda^{2} p(p-1) / 2-1>0$.

Note that, since we chose $H \in\left(\frac{1}{2}+\lambda^{2} / 2,1\right)$, then ( $\left.\mathscr{H}(2)\right)$ is always satisfied.

We first state two useful results.

Proposition 1. Let $\left(\mathcal{F}_{l}\right)_{l>0}$ be the filtration

$$
\mathcal{F}_{l}=\sigma\left\{\left(\varepsilon_{l^{\prime}}(u), \omega_{l^{\prime}}(u)\right), u \in \mathbb{R}, l^{\prime} \geq l\right\} .
$$

Then, for fixed $t>0,\left(X_{l}(t), l>0\right)$ is an $\mathcal{F}_{l}$-martingale.

Proof. This is a straightforward application of Properties 2 and 3.

Proposition 2. Let $p \geq 2$ be an integer. Then, for $t \in[0, T]$,

$$
\lim _{l \rightarrow 0} \mathrm{E}\left[X_{l}(t)^{p}\right]=K(p) t^{p H-\lambda^{2} p(p-1) / 2},
$$

where $K(p) \in(0,+\infty]$ is a constant that depends on the parameters $\sigma^{2}, T, \lambda^{2}$, and $H$, but not on $t$. Moreover, $K(p)$ is finite if and only if $p$ satisfies $(\mathcal{H}(p))$.

The proof of Proposition 2 is postponed until Appendix B, where the reader can also find the value of the constant $K(p)$.

It is then easy to prove the following result.

Theorem 1. For fixed $t \geq 0, X_{l}(t)$ goes to a nondegenerate limit $X(t)$ as $l$ goes to 0 , and the convergence holds almost surely and in $L^{3}$. Moreover, the process $X=(X(t), t \geq 0)$ is well defined as a continuous version of the almost-sure limit of $\left(X_{l}(t), t \geq 0\right)$ in the space of continuous functions.

Proof. Applying Proposition 1 and a classical result from the theory of martingales, if, for some fixed $t>0$, the moments $\mathrm{E}\left[\left|X_{l}(t)\right|^{p}\right]$ remain bounded for some $p>1$, we have

$$
X(t)=\lim X_{l}(t) \quad \text { as } l \rightarrow 0
$$

almost surely and in $L^{q}$ for all $q \in[1, p)$. Since we choose $H>\frac{1}{2}+\lambda^{2} / 2$ and $\lambda^{2}<\frac{1}{4}$, ( $\left.\mathscr{H}(4)\right)$ holds. The first half of the statement of the theorem then follows from Proposition 2. 
Moreover, it also follows from Proposition 2 that the Kolmogorov criterion for convergence and regularity of stochastic processes is satisfied: there exists some $a>0, b>0$, and $c>0$ such that, for any $t \in[0, T]$ and any $l>0$ small enough,

$$
\mathrm{E}\left[\left|X_{l}(t)\right|^{a}\right] \leq c t^{1+b} .
$$

Indeed, we can choose $a=4$ and $b=4 H-6 \lambda^{2}-1$. The rest of the theorem follows from a standard application of this criterion.

Remark. From Properties 1 and $3, X$ is a process with stationary increments and zero expectation.

\subsection{Explicit construction of $(\varepsilon, \omega)$}

The construction of the process $\omega$ is almost the same as that used in the definition of the symmetrical MRW in [2]. The process $\varepsilon$ is also constructed in a similar fashion. Let us consider the 'time scale' half-plane $\mathbb{R} \times(0,+\infty)$, and define on it a two-dimensional Gaussian white noise $P\left(\mathrm{~d} t^{\prime}, \mathrm{d} l^{\prime}\right)$ with variance $l^{\prime-2} \mathrm{~d} t^{\prime} \times \mathrm{d} l^{\prime}$. Then $\omega$ is obtained as

$$
\omega_{l}(t)=-\frac{\lambda^{2}}{2(\log (T / l)+1)}+\lambda \int_{\mathcal{A}_{l}(t)} P\left(\mathrm{~d} t^{\prime}, \mathrm{d} l^{\prime}\right),
$$

where $\mathcal{A}_{l}(t)$ is the conical domain

$$
\mathcal{A}_{l}(t)=\left\{\left(t^{\prime}, l^{\prime}\right) \in \mathbb{R} \times(0,+\infty), l^{\prime} \geq l, 0 \leq t-t^{\prime} \leq \min \left(l^{\prime}, T\right)\right\} .
$$

So, to construct $\varepsilon$, we now consider the domain

$$
\mathcal{B}_{l}(t)=\left\{\left(t^{\prime}, l^{\prime}\right) \in \mathbb{R} \times(0,+\infty), l^{\prime} \geq l, 0 \leq t^{\prime}-t \leq l^{\prime}\right\}
$$

and define

$$
\varepsilon_{l}(t)=\sigma \int_{\mathcal{B}_{l}(t)} l^{\prime-1+H} P\left(\mathrm{~d} t^{\prime}, \mathrm{d} l^{\prime}\right) .
$$

We refer the reader to Figure 1 for a graphical representation of $\mathcal{A}_{l}(t)$ and $\mathscr{B}_{l}(t)$. In Appendix A we give the exact first and second moments of $(\varepsilon, \omega)$, which are obtained through straightforward computations. It is then easy to check that $(\varepsilon, \omega)$ satisfies Properties 1 to 5 . In particular, the constants $c^{\varepsilon}$ and $c^{\omega \varepsilon}$ of Property 4 are respectively equal to $1 /(2-2 H)(3-2 H)$ and $\left(2^{2-H}-2\right) /(1-H)(2-H)$.

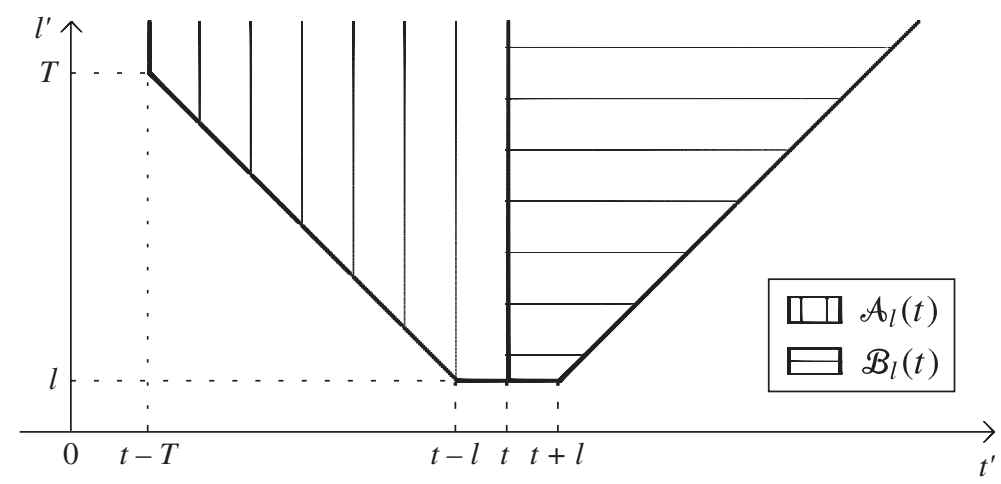

Figure 1: The cones $\mathcal{A}_{l}(t)$ and $\mathscr{B}_{l}(t)$. 


\section{Scaling and moments of the skewed process $X$}

The following theorem characterizes the scaling behavior of the distribution of $X(t)$.

Theorem 2. For $r \in(0,1]$,

$$
(X(r t), 0 \leq t \leq T) \stackrel{\text { law }}{=} \mathrm{e}^{\Omega_{r}} r^{H}(X(t), 0 \leq t \leq T),
$$

where $\Omega_{r} \sim N\left(-\lambda^{2} \ln \left(r^{-1}\right) / 2, \lambda^{2} \ln \left(r^{-1}\right)\right)$ is a Gaussian constant independent of $X$.

Proof. First note that it follows from Properties 3 and 5 that, for fixed $l>0$ and $r \in(0,1]$,

$$
\left(\left(\varepsilon_{r l}(r u), \omega_{r l}(r u)\right), u \in[0, T]\right) \stackrel{\text { law }}{=}\left(\left(r^{-1+H} \varepsilon_{l}(u), \Omega_{r}+\omega_{l}(u)\right), u \in[0, T]\right),
$$

where $\Omega_{r}$ is an $N\left(-\lambda^{2} \ln \left(r^{-1}\right) / 2, \lambda^{2} \ln \left(r^{-1}\right)\right)$ random variable, which is furthermore independent of $(\varepsilon, \omega)$. From this we deduce that

$$
\left(\varepsilon_{r l}(r u) \exp \left(\omega_{r l}(r u)\right), u \in[0, T]\right) \stackrel{\text { law }}{=} r^{-1+H} \exp \left(\Omega_{r}\right)\left(\varepsilon_{l}(u) \exp \left(\omega_{l}(u)\right), u \in[0, T]\right) .
$$

We now consider the process $X_{r l}(r t)$ :

$$
\begin{aligned}
\left(X_{r l}(r t), t \in[0, T]\right) & =\left(\int_{0}^{r t} \varepsilon_{r l}(u) \exp \left(\omega_{r l}(u)\right) \mathrm{d} u, t \in[0, T]\right) \\
& =\left(r \int_{0}^{t} \varepsilon_{r l}(r u) \exp \left(\omega_{r l}(r u)\right) \mathrm{d} u, t \in[0, T]\right) \\
& \stackrel{\text { law }}{=} r^{H} \exp \left(\Omega_{r}\right)\left(\int_{0}^{t} \varepsilon_{l}(u) \exp \left(\omega_{l}(u)\right) \mathrm{d} u, t \in[0, T]\right) \\
& =r^{H} \exp \left(\Omega_{r}\right)\left(X_{l}(t), t \in[0, T]\right) .
\end{aligned}
$$

Taking the limit $l \rightarrow 0$ gives (4).

We now turn to the absolute moments of $X$, and show that they satisfy (2) with an exact equality.

Theorem 3. If, for some $q>0$, there is an even integer $p>q$ such that $(\mathcal{H}(p))$ is satisfied, then, for $t \in[0, T]$,

$$
\mathrm{E}\left[|X(t)|^{q}\right]=C(q) t^{q H-\lambda^{2} q(q-1) / 2},
$$

where $C(q)$ is the positive finite constant

$$
C(q)=T^{-q H+q(q-1) \lambda^{2} / 2} \mathrm{E}\left[|X(T)|^{q}\right] .
$$

If $q$ is moreover an integer then

$$
\mathrm{E}\left[X(t)^{q}\right]=K(q) t^{q H-\lambda^{2} q(q-1) / 2}
$$

where $K(q)$ is the same as in Proposition 2. Conversely, if $(\mathscr{H}(q))$ is not satisfied for some $q>2, \mathrm{E}\left[|X(t)|^{q}\right]=+\infty$ for $t>0$. 
Proof. It follows from Propositions 1 and 2 that $X_{l}(T)$ converges in $L^{q}$ to $X(T)$, so $\mathrm{E}\left[|X(T)|^{q}\right]$ is finite. In the case where $q$ is an integer, (5) is also a direct consequence of these two propositions. In the general case, we apply Theorem 2: by setting $r=t / T$, we have

$$
\mathrm{E}\left[|X(t)|^{q}\right]=T^{-q H+q(q-1) \lambda^{2} / 2} \mathrm{E}\left[|X(T)|^{q}\right] t^{q H-\lambda^{2} q(q-1) / 2} .
$$

Conversely, let us suppose that $\mathrm{E}\left[|X(t)|^{q}\right]$ is finite for some $t \in(0, T]$ and $q>1$. Then, from the stationarity of the increments of $X$ and a basic convexity inequality, we obtain

$$
\mathrm{E}\left[|X(t)|^{q}\right]>2 \mathrm{E}\left[\left|X\left(\frac{t}{2}\right)\right|^{q}\right] .
$$

Then, applying Theorem 2 , we have $2^{1-q H+\lambda^{2} q(q-1) / 2}<1$, so $(\mathscr{H}(q))$ is satisfied. This proves the result.

\section{Modeling the asymmetry of financial data}

\subsection{Preliminaries}

In this section we focus on the second- and third-order properties of the increments of the process $X$, and show how, depending on the value of the parameter $H$, they can reflect the following two stylized facts. First, there is no statistically significant correlation between two log-returns at different times. Second, there is a negative, slightly significant correlation between the past log-returns and the future squared log-returns, while the converse is false: past volatilities and future returns appear to be uncorrelated. (Note that while the former fact is universally observed in financial asset prices, the latter is mainly observed in stocks and index prices; see [5].)

Let us examine the moment of the second order of $X$.

Proposition 3. For $t \in[0, T]$,

$$
\mathrm{E}\left[X(t)^{2}\right]=\frac{2 \sigma^{2} T^{\lambda^{2}} c^{\varepsilon}}{\left(2 H-1-\lambda^{2}\right)\left(2 H-\lambda^{2}\right)} t^{2 H-\lambda^{2}} .
$$

Proof. This is a simple application of Proposition 2 and of the value of $K(2)$ given in Appendix B.

Let us now recall that $X$ is properly defined only in the case $H \in\left(\frac{1}{2}+\lambda^{2} / 2,1\right)$ (so that $(\mathscr{H}(4))$ holds). Thus, we can write $\mathrm{E}\left[X(t)^{2}\right]=K(2) t^{1+d}$ with $K(2)$ being the above fraction and

$$
d=2 H-1-\lambda^{2}>0 \text {. }
$$

So as to obtain a satisfying model of financial data, we clearly have to place ourselves in a regime where $d$ is small, so that $\mathrm{E}\left[X(t)^{2}\right]$ scales approximately as a linear function of $t$, and the covariance between the increments of $X$ at different times vanishes. However, as can be seen from Proposition 3, $K(2)$ goes to $+\infty$ as $d$ goes to 0 .

In this section we therefore study the regime of small $d$, and the second- and third-order properties of the normalized process:

$$
Y_{d}(t)=-\sigma \frac{X(t)}{\left(\mathrm{E}\left[X(1)^{2}\right]\right)^{1 / 2}} .
$$


Note that we introduced a minus sign so as to reproduce the negative skewness empirically observed, and we added a subscript $d$ to emphasize the dependence on this parameter (we will continue to use the notation $d \in\left(0,1-\lambda^{2}\right)$ instead of $\left.H \in\left(\frac{1}{2}+\lambda^{2} / 2,1\right)\right)$. We intend to show here that, for a well-chosen value of $d$, the process $Y_{d}$ reproduces the type of asymmetry observed in stocks and index prices.

Recall that the notation $\delta_{\tau} Y_{d}(t)$ refers to the increment $Y_{d}(t+\tau)-Y_{d}(t)$. For $\tau>0$ and $k \in \mathbb{Z}$, we are interested in the functions

$$
\rho_{d}^{(1)}(\tau, k)=\frac{\mathrm{E}\left[\delta_{\tau} Y_{d}(0) \delta_{\tau} Y_{d}(k \tau)\right]}{\mathrm{E}\left[\delta_{\tau} Y_{d}(0)^{2}\right]}
$$

and

$$
\rho_{d}^{(2)}(\tau, k)=\frac{\mathrm{E}\left[\delta_{\tau} Y_{d}(0) \delta_{\tau} Y_{d}(k \tau)^{2}\right]}{\left(\mathrm{E}\left[\delta_{\tau} Y_{d}(0)^{2}\right]\right)^{2}},
$$

where the normalization of $\rho^{(2)}$ was introduced in [6], and used in, for instance, [9] and [16]. Alternatively, we could examine the proper linear correlation between $\delta_{\tau} Y_{d}(0)$ and $\delta_{\tau} Y_{d}(k \tau)^{2}$, that is,

$$
\rho_{d}^{(3)}(\tau, k)=\frac{\mathrm{E}\left[\delta_{\tau} Y_{d}(0) \delta_{\tau} Y_{d}(k \tau)^{2}\right]}{\left(\mathrm{E}\left[\delta_{\tau} Y_{d}(0)^{2}\right]\right)^{1 / 2}\left(\mathrm{E}\left[\delta_{\tau} Y_{d}(0)^{4}\right]\right)^{1 / 2}} .
$$

Since we are dealing with correlations that empirically decay to 0 after a few lags, we will restrict ourselves to the case $(|k|+1) \tau \leq T$.

\subsection{Behavior of $Y_{d}$ in the regime of small $d$}

We first examine the moments of $Y_{d}$ as $d$ goes to 0 .

Proposition 4. For $t \in[0, T]$ and $p \geq 2$, an even integer such that $\mathrm{E}\left[X(t)^{p}\right]<+\infty$, $\mathrm{E}\left[Y_{d}(t)^{p}\right]$ remains positive and bounded as d goes to 0 . However, if $p$ is odd, $\mathrm{E}\left[Y_{d}(t)^{p}\right]$ goes to 0 .

Proof. Again, this is a direct consequence of Proposition 2 and of the value of $K(p)$ given in Appendix B.

Remark. Proposition 4 suggests that a limiting process

$$
Y(t)=\lim Y_{d}(t) \quad \text { as } d \rightarrow 0
$$

may exist, where the convergence is understood as a convergence in distribution. This is quite reminiscent of the study by Arby et al. [1], who investigated the validity of the limit

$$
\lim \frac{\int_{0}^{t} \mathrm{e}^{\omega_{l}}(u) \mathrm{d} B^{H}(u)}{\mathrm{E}\left[\left(\int_{0}^{t} \mathrm{e}^{\omega_{l}}(u) \mathrm{d} B^{H}(u)\right)^{2}\right]^{1 / 2}} \quad \text { as } l \rightarrow 0,
$$

where $B^{H}$ is a fractional Brownian motion with Hurst exponent $H$, which is (in contrast with our setting) independent of $\omega$. In the case $H=\frac{1}{2}+\lambda^{2} / 2$ (that is, $d=0$ in our notation), these authors obtained only the convergence of the moments of integer order and postulated the convergence in law. Note however that in the present work, we are not chiefly interested in the validity of the convergence $Y_{d} \rightarrow Y$, since the moments of order three of $Y_{d}$ vanish, so that the limiting process $Y_{0}$ (if it exists) has a skewness equal to 0 . 
We now place ourselves in the regime of small but nonzero values of $d$, and examine the magnitude of the correlation functions $\rho_{d}^{(i)}, i=1,2,3$.

Theorem 4. For $0<\tau<T$ and $(|k|+1) \tau \leq T$, we have

$$
\begin{gathered}
\rho_{d}^{(1)}(\tau, k)=O(d) \quad \text { if }|k| \geq 1, \\
\left|\rho_{d}^{(2)}(\tau, k)\right|=O\left(d^{1 / 2}\right) \quad \text { and } \quad\left|\rho_{d}^{(3)}(\tau, k)\right|=O\left(d^{1 / 2}\right) \quad \text { if } k \geq 0, \\
\left|\rho_{d}^{(2)}(\tau, k)\right|=O\left(d^{3 / 2}\right) \quad \text { and }\left|\rho_{d}^{(3)}(\tau, k)\right|=O\left(d^{3 / 2}\right) \quad \text { if } k<0,
\end{gathered}
$$

as $d \rightarrow 0$.

Proof. See Appendix C.

Theorem 4 therefore suggests that, when $d$ is of order roughly 0.01 to $0.1, \rho_{d}^{(2)}(\tau, k)$ for $k \geq 0$ is significantly nonzero (as it is of order $d^{1 / 2}$ ), while $\rho_{d}^{(2)}(\tau, k)$ for $k<0$ and $\rho_{d}^{(1)}(\tau, k)$ for $k \neq 0$ are much smaller, and in practice indiscernible from the noise. We refer the reader to Subsection 6.2 for an empirical discussion concerning the choice of $d$.

\section{Numerical simulation and comparison to real data}

In this section we present a numerical method for simulating the process we introduce, and we compare the leverage effect observed in simulations to the leverage effect measured from empirical data. Since the main objective of this paper is to define and study the mathematical properties of the model, we do not discuss any parameter estimation issue. This problem and a more exhaustive comparison to financial data will be the subject of a forthcoming work.

\subsection{The simulation scheme}

We propose in this section to approximate the increments

$$
\delta_{\tau} X(k \tau)=\lim _{l \rightarrow 0} \int_{k \tau}^{(k+1) \tau} \varepsilon_{l}(u) \mathrm{e}^{\omega_{l}(u)} \mathrm{d} u
$$

of the process $X$ (for $k \in \mathbb{N}$ and $\tau>0$ ) by Riemann sums. If the parameter $d=2 H-1-\lambda^{2}$ defined in the previous section is large enough, this is easily done; however, if this parameter is small then some extra difficulties must be taken care of. This comes mainly from the fact that the approximation

$$
\frac{1}{n} \sum_{k=1}^{n}\left(\frac{k}{n}\right)^{-1+d} \approx \int_{0}^{1} u^{-1+d} \mathrm{~d} u
$$

is valid only in a regime $n \gg \mathrm{e}^{1 / d}$, which might be unfeasible in practice.

We set $\left(l_{n}, n \in \mathbb{N}\right)$ as

$$
l_{n}=\left(d\left(1-\frac{d}{2}-\frac{\lambda^{2}}{2}\right)\right)^{1 /(1-d)} n^{-1}
$$

and

$$
\delta_{\tau} \tilde{X}_{1 / n}(k \tau)=n^{-1} \sum_{j=\lfloor k \tau n\rfloor}^{\lfloor n(k+1) \tau\rfloor-1} \varepsilon_{l_{n}}\left(\frac{j}{n}\right) \mathrm{e}^{\omega_{l_{n}}(j / n)},
$$

where $(\varepsilon, \omega)$ is as in Subsection 3.3. We then have the following result. 
Theorem 5. For $k \in \mathbb{N}$ and $\tau>0, \delta_{\tau} \tilde{X}_{1 / n}(k \tau)$ converges to $\delta_{\tau} \tilde{X}(k \tau)$ in $L^{2}$ as $n \rightarrow+\infty$. Moreover, let us define

$$
r_{n}=\frac{\left|\mathrm{E}\left[\left(\delta_{\tau} X(k \tau)\right)^{2}\right]-\mathrm{E}\left[\left(\delta_{\tau} \tilde{X}_{1 / n}(k \tau)\right)^{2}\right]\right|}{\mathrm{E}\left[\left(\delta_{\tau} X(k \tau)\right)^{2}\right]} .
$$

Then $r_{n}$ is of order $d n^{-d}$, that is, for fixed $n, r_{n} / d$ is bounded as $d \rightarrow 0$, and, for fixed $d, n^{d} r_{n}$ is bounded as $d \rightarrow+\infty$.

Proof. Without loss of generality, we suppose that $\tau=1$. The exact value of $\mathrm{E}\left[\left(\delta_{1} X(k)\right)^{2}\right]$ can be found in Proposition 3; we rewrite it as

$$
\mathrm{E}\left[\left(\delta_{1} X(k)\right)^{2}\right]=2 \sigma^{2} T^{\lambda^{2}} c^{\varepsilon}\left(\frac{n^{-d}}{d}+\int_{1 / n}^{1} u^{-1+d} \mathrm{~d} u-\int_{0}^{1} u^{d} \mathrm{~d} u\right) .
$$

In order to compute $\mathrm{E}\left[\left(\delta_{1} \tilde{X}_{1 / n}(k)\right)^{2}\right]$, we use the relation

$$
\begin{aligned}
& \varepsilon_{l_{n}}\left(\frac{k_{1}}{n}\right) \varepsilon_{l_{n}}\left(\frac{k_{2}}{n}\right) \exp \left(\omega_{l_{n}}\left(\frac{k_{1}}{n}\right)+\omega_{l_{n}}\left(\frac{k_{2}}{n}\right)\right) \\
& =\left.\frac{\partial^{2}}{\partial x_{1} \partial x_{2}}\right|_{x_{1}=x_{2}=0} \exp \left(\omega_{l_{n}}\left(\frac{k_{1}}{n}\right)+\omega_{l_{n}}\left(\frac{k_{2}}{n}\right)+x_{1} \varepsilon_{l_{n}}\left(\frac{k_{1}}{n}\right)+x_{2} \varepsilon_{l_{n}}\left(\frac{k_{2}}{n}\right)\right) .
\end{aligned}
$$

This and the values of the covariance functions given in Appendix A yield

$$
\mathrm{E}\left[\left(\delta_{1} \tilde{X}_{1 / n}(k)\right)^{2}\right]=\frac{\sigma^{2} T^{\lambda^{2}} c^{\varepsilon}}{n}\left(\left(2-d-\lambda^{2}\right) l_{n}^{-1+d}+2 \sum_{k=1}^{n-1}\left(1-\frac{k}{n}\right)\left(\frac{k}{n}\right)^{-1+d}\right) .
$$

Using a first-order Taylor expansion gives the result for $r_{n}$.

Moreover, following the same lines, we easily obtain the exact value of $\mathrm{E}\left[\delta_{1} X(k) \delta_{1} \tilde{X}_{1 / n}(k)\right]$. This allows us to check that $\mathrm{E}\left[\left(\delta_{1} X(k)-\delta_{1} \tilde{X}_{1 / n}(k)\right)^{2}\right]$ goes to 0 as $n$ goes to $+\infty$.

Remark. If $l_{n}$ is not chosen as the value that we specify above, but instead as a more generic value like $l_{n}=1 / n$, then $r_{n}$ will be of order $n^{-d}$, which may decrease very slowly to 0 for small $d$.

Theorem 5 shows that we can well approximate the increments of the process $X$ through the discrete process $\tilde{X}_{1 / n}$. This last process is easily simulated with the help of some efficient procedures for the simulation of stationary Gaussian random fields, such as that proposed in [8], which is based on fast Fourier transforms.

\subsection{Numerical results and comparisons to empirical data}

Let us illustrate the previous results with some numerical simulations. We choose $\sigma^{2}=1$, $\lambda^{2}=0.04$, and $T=200$, which are usual values for modeling financial data with the MRW model (see [3]). The parameter $d$ has been chosen to vary from 0.01 to 0.3 , i.e. $H$ varies from 0.525 to 0.67 . In all the reported results, we set $\tau=1, N=5000$, and $n=500$, and we performed averages over 100 realizations of the process. For each value of $d$, each realization of the sequence $\delta_{1} \tilde{X}_{1 / n}(0), \ldots, \delta_{1} \tilde{X}_{1 / n}(N-1)$ has been simulated using the techniques described above. We then approximated $\delta_{1} Y_{d}(k)$ by

$$
\delta_{1} Y_{d}(k) \approx-\frac{\delta_{1} \tilde{X}_{1 / n}(k)}{\left(\left(\delta_{1} \tilde{X}_{1 / n}\right)^{2}\right)^{1 / 2}},
$$

$\overline{\left(\delta_{1} \tilde{X}_{1 / n}\right)^{2}}$ being the empirical means of the squared increments $\delta_{1} \tilde{X}_{1 / n}(0), \ldots, \delta_{1} \tilde{X}_{1 / n}(N-1)$. 


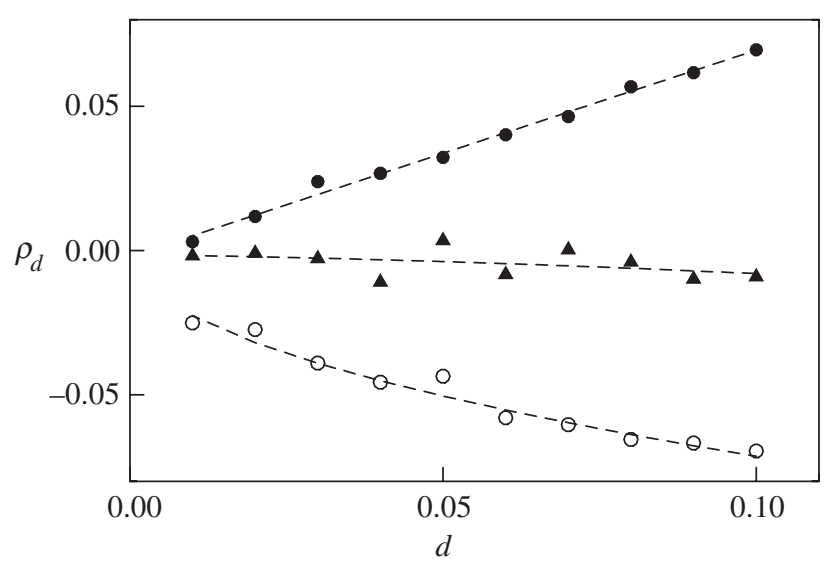

Figure 2: Values $\rho_{d}^{(1)}(1)(\bullet), \rho_{d}^{(3)}(1)(\circ)$, and $\rho_{d}^{(3)}(-1)(\mathbf{\Delta})$ for $d=0.01$ to $d=0.10$, and their respective adjustment to the fits $c_{1} d, c_{2} d^{1 / 2}$, and $c_{3} d^{3 / 2}$ (dashed lines).

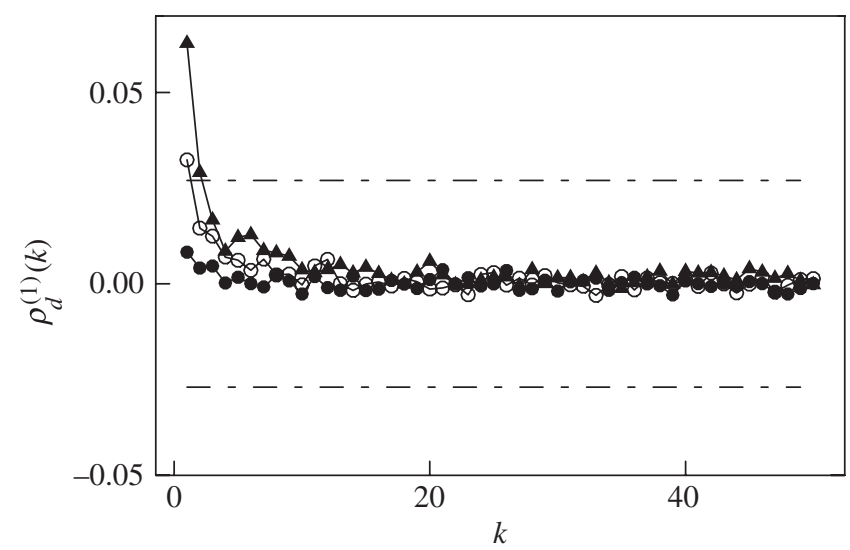

FIGURE 3: Correlations $\rho_{d}^{(1)}(k)$ for $1 \leq k \leq 50$ with $d=0.01(\bullet), d=0.05(\circ)$, and $d=0.10(\boldsymbol{\Lambda})$. The dashed lines represent the $95 \%$ interval for uncorrelated random variables for a series of size 5000 .

In Figure 2 we check the dependency of the correlation functions $\rho_{d}^{(1)}$ and $\rho_{d}^{(3)}$ (defined in (6) and (7)) on the parameter $d$. Recall that we obtained

$$
\begin{gathered}
\rho_{d}^{(1)}(k)=O(d) \quad \text { for all }|k| \geq 1, \quad \rho_{d}^{(3)}(k)=O\left(d^{1 / 2}\right) \quad \text { for all } k \geq 0 \\
\text { and } \quad \rho_{d}^{(3)}(k)=O\left(d^{3 / 2}\right) \quad \text { for all } k \leq-1 .
\end{gathered}
$$

These are well confirmed by our simulations.

In Figure 3 we plot the autocorrelations $\rho_{d}^{(1)}(k)$ of the return series as a function of the lag $k$ for $d=0.01,0.05$, and 0.1 . We see that after a few lags all series are almost uncorrelated; but it is only for small enough $d(d \leq 0.05)$ that the first lag correlation is inside the $95 \%$ confidence interval of a series of $N=5000$ uncorrelated random variables. Since financial returns are well known to be uncorrelated (or very weakly correlated), the parameter $d$ should probably be chosen below the value 0.05 . 


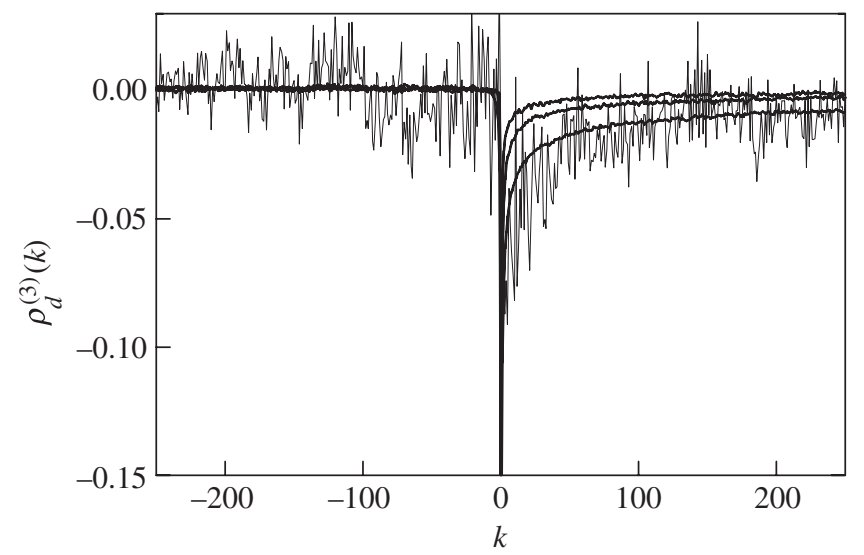

FIGURE 4: Correlations $\rho_{d}^{(3)}(k)$ for $|k| \leq 250$. The thick lines represent, in order from top to bottom, $d=0.03, d=0.1$, and $d=0.30$. The noisy curve corresponds to real data estimated on a basket of five indices and is shown for comparison.
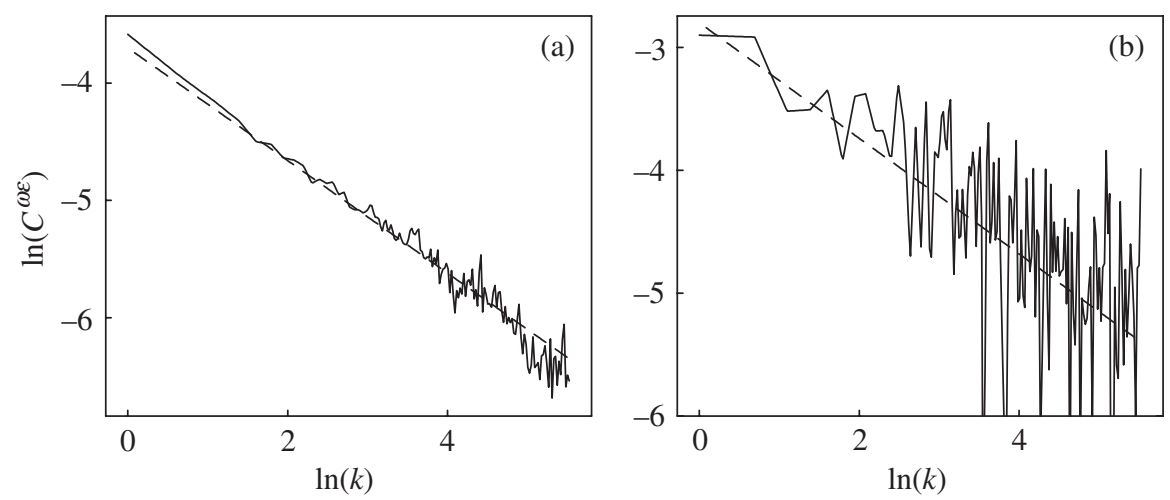

FIGURE 5: Plot of $C^{\omega \varepsilon}=-\operatorname{corr}\left[\delta_{1} p(0), 2 \log \left(\left|\delta_{1} p(k \tau)\right|\right)\right]$ for $|k| \leq 50$ on a $\log$-log scale. (a) Estimated correlation for 100 realizations of a skewed MRW with $\lambda^{2}=0.04, T=250$, and $d=0.03$. (b) Same graph for the mean correlations over five indices. The dashed lines represent the expected power-law behavior $c k^{\left(-1+\lambda^{2}+d\right) / 2} \sim k^{-0.48}$.

In Figure 4 we report the estimation of the leverage effect on our simulated series. We estimate $\rho_{d}^{(3)}(k)$ as a function of $k$ for three values of $d$. For comparison purposes, we also plot the correlation that we measure on real data, namely the daily quotation of five stock indices. More precisely, we considered the CAC 40, DAX, FTSE 100, S\&P 500, and Dow Jones index daily series from 1990/12/03 to 2010/02/15 and averaged the empirical correlations over the five indices, so as to reduce the noise. We confirm our previous computations: the estimated function $\rho_{d}^{(3)}(k)$ in our simulation exhibits a strong asymmetry and is clearly negative for positive lags $k$. Moreover, we see that, as $d$ increases, the leverage effect indeed becomes stronger. The curves we obtain seem quite similar to the effect observed on stock index returns.

Finally, in Figure 5 we present another way to assess the leverage effect. Indeed, the construction of our model directly suggests that there should exist a negative correlation between past returns and the logarithm of future volatilities, and this correlation should behave as a power law of the time lag. That is, if we denote by $p(t)$ the log-price of an asset then the following 
relation is expected:

$$
C^{\omega \varepsilon}(k) \equiv-\operatorname{corr}\left[\delta_{\tau} p(0), 2 \log \left(\left|\delta_{\tau} p(k \tau)\right|\right)\right] \sim c k^{-\alpha}
$$

for $k \geq 1$, some constant $c>0$, and some exponent $\alpha \in(0,1)$. From the definition of $\gamma^{\omega \varepsilon}$, we expect to find in our model

$$
\alpha \approx-1+H=\frac{-1+\lambda^{2}+d}{2},
$$

which is close to $\frac{1}{2}$. Figure 5(a) shows that this is indeed the case. We have

$$
C^{\omega \varepsilon}(k) \simeq \gamma^{\omega \varepsilon}(k) \sim c k^{\left(-1+\lambda^{2}+d\right) / 2} .
$$

In the case of real data, as illustrated in Figure 5(b), we observe that a power law with exponent $\alpha \simeq 0.48$ provides a good fit to the data.

\section{Appendix A. Covariances of $\omega$ and $\varepsilon$}

Through straightforward computations, it is possible to obtain the following covariance functions for $(\varepsilon, \omega)$. Fix $0<l^{\prime}<l<T$ and $u, \tau \in \mathbb{R}$. The process $\omega$ has expectation

$$
\mathrm{E}\left[\omega_{l}(u)\right]=-\frac{\lambda^{2}}{2}\left(\log \left(\frac{T}{l}\right)+1\right)
$$

and covariance

$$
\operatorname{cov}\left[\omega_{l}(u), \omega_{l^{\prime}}(u+\tau)\right]=\gamma_{l}^{\omega}(\tau)
$$

with

$$
\gamma_{l}^{\omega}(\tau)= \begin{cases}\lambda^{2}\left(\log \left(\frac{T}{l}\right)+1-\frac{\tau}{l}\right) & \text { if }|\tau| \leq l, \\ \lambda^{2} \log \left(\frac{T}{\tau}\right) & \text { if } l \leq|\tau| \leq T, \\ 0 & \text { if } T \leq|\tau| .\end{cases}
$$

The process $\varepsilon$ has zero expectation and satisfies

$$
\operatorname{cov}\left[\varepsilon_{l}(u), \varepsilon_{l^{\prime}}(u+\tau)\right]=\gamma_{l}^{\varepsilon}(\tau),
$$

where $\gamma_{l}^{\varepsilon}(\tau)$ is defined as

$$
\gamma_{l}^{\varepsilon}(\tau)= \begin{cases}\frac{\sigma^{2}}{(2-2 H)(3-2 H)}|\tau|^{-2+2 H} & \text { if } l \leq|\tau|, \\ \sigma^{2}\left(\frac{1}{2-2 H}-\frac{1}{3-2 H} \frac{|\tau|}{l}\right) l^{-2+2 H} & \text { if } 0 \leq|\tau| \leq l .\end{cases}
$$

Finally, the covariance between $\varepsilon$ and $\omega$ is given by

$$
\operatorname{cov}\left[\varepsilon_{l}(u), \omega_{l^{\prime}}(u+\tau)\right]=\operatorname{cov}\left[\varepsilon_{l^{\prime}}(u), \omega_{l}(u+\tau)\right]=\gamma_{l}^{\omega \varepsilon}(\tau),
$$


where $\gamma_{l}^{\omega \varepsilon}(\tau)$ is defined as

$$
\gamma_{l}^{\omega \varepsilon}(\tau)= \begin{cases}0 & \text { if } \tau<0, \\ \frac{\lambda \sigma}{2-H} \tau l^{-2+H} & \text { if } 0 \leq \tau \leq l, \\ \lambda \sigma\left(\frac{2}{1-H} l^{-1+H}-\frac{1}{2-H} \frac{\tau}{l} l^{-1+H}\right. & \\ \left.-\frac{2}{(1-H)(2-H)} \tau^{-1+H}\right) & \text { if } l \leq \tau \leq 2 l, \\ \frac{\lambda \sigma}{(1-H)(2-H)}\left(2^{2-H}-2\right) \tau^{-1+H} & \text { if } 2 l \leq \tau \leq T, \\ \frac{\lambda \sigma}{(1-H)(2-H)}\left(\left(2^{2-H}-1\right) \tau^{-1+H}-T^{-1+H}\right) & \text { if } T \leq \tau \leq 2 T, \\ \frac{\lambda \sigma}{(1-H)(2-H)}\left((\tau-T)^{-1+H}-\tau^{-1+H}\right) & \text { if } \tau \geq 2 T .\end{cases}
$$

\section{Appendix B. Proof of Proposition 2}

We begin by evaluating the moment $\mathrm{E}\left[X_{l}(t)^{p}\right]$ for $l \in(0, T), t \geq 0$, and $p \geq 2$. From Fubini's theorem we have

$$
\begin{aligned}
& \mathrm{E}\left[\left(\int_{0}^{t} \varepsilon_{l}(u) \mathrm{e}^{\omega_{l}(u)} \mathrm{d} u\right)^{p}\right] \\
& \quad=\int_{0}^{t} \cdots \int_{0}^{t} \mathrm{~d} u_{1} \cdots \mathrm{d} u_{p} \mathrm{E}\left[\varepsilon_{l}\left(u_{1}\right) \cdots \varepsilon_{l}\left(u_{p}\right) \exp \left(\omega_{l}\left(u_{1}\right)+\cdots+\omega_{l}\left(u_{p}\right)\right)\right] .
\end{aligned}
$$

We compute the right-hand side using the relation

$$
\begin{aligned}
\varepsilon_{l}\left(u_{1}\right) & \cdots \varepsilon_{l}\left(u_{p}\right) \exp \left(\omega_{l}\left(u_{1}\right)+\cdots+\omega_{l}\left(u_{p}\right)\right) \\
& =\left.\frac{\partial^{p}}{\partial x_{1} \cdots \partial x_{p}}\right|_{x_{1}=\cdots=x_{p}=0} \exp \left(\omega_{l}\left(u_{1}\right)+\cdots+\omega_{l}\left(u_{p}\right)+x_{1} \varepsilon_{l}\left(u_{1}\right)+\cdots+x_{p} \varepsilon_{l}\left(u_{p}\right)\right) .
\end{aligned}
$$

Permuting the expectation and differentiation, we differentiate $p$ times:

$$
\mathrm{E}\left[\exp \left(\omega_{l}\left(u_{1}\right)+\cdots+\omega_{l}\left(u_{p}\right)+x_{1} \varepsilon_{l}\left(u_{1}\right)+\cdots+x_{p} \varepsilon_{l}\left(u_{p}\right)\right)\right]=\exp \left(S_{p}\left(x_{1}, \ldots, x_{p}\right)\right) .
$$

The term $S_{p}=S_{p}\left(x_{1}, \ldots, x_{p}\right)$ can be evaluated as

$$
\begin{aligned}
S_{p}= & \sum_{1 \leq i<j \leq p} \gamma_{l}^{\omega}\left(u_{i}-u_{j}\right)+\sum_{1 \leq i, j \leq p} x_{j} \gamma_{l}^{\omega \varepsilon}\left(u_{i}-u_{j}\right) \\
& +\sum_{1 \leq i<j \leq p} x_{i} x_{j} \gamma_{l}^{\varepsilon}\left(u_{i}-u_{j}\right)+\frac{1}{2} \sum_{i=1}^{p} x_{i}^{2} \gamma_{l}^{\varepsilon}(0),
\end{aligned}
$$

where we have used Property 3. From Property $4, \gamma_{l}^{\omega \varepsilon}\left(u_{i}-u_{j}\right)$ is nonzero if and only if $u_{i}>u_{j}$; however, this will not be used in what follows: we do not keep track of the order of the $u_{i}$ s so as to avoid introducing notation that would be of no use in this proof. 
We will however need the following definitions: for $i, j=1, \ldots, p$,

$$
\begin{aligned}
D_{i} & =D_{i}\left(x_{1}, \ldots, x_{p}\right)=\frac{\partial}{\partial x_{i}} S_{p}\left(x_{1}, \ldots, x_{p}\right), \\
D_{i, j} & =D_{i, j}\left(x_{1}, \ldots, x_{p}\right)=\frac{\partial}{\partial x_{j}} D_{i}\left(x_{1}, \ldots, x_{p}\right),
\end{aligned}
$$

and, for $1 \leq n \leq p$,

$$
R_{n}=R_{n}\left(x_{1}, \ldots, x_{p}\right)=\frac{\partial^{n}}{\partial x_{1} \cdots x_{n}} \mathrm{e}^{S_{p}\left(x_{1}, \ldots, x_{p}\right)} .
$$

Also, for $1 \leq n \leq p$ and $0 \leq m \leq\lfloor n / 2\rfloor$, we define $E_{m, n}$ to be the set of all partitions $P$ of $\{1, \ldots, n\}$ into $n-m$ subsets such that $m$ of these subsets have two elements and the other $n-2 m$ subsets have one element:

$$
E_{m, n}=\left\{P=\left\{\left\{a_{1}, a_{2}\right\}, \ldots,\left\{a_{2 m-1}, a_{2 m}\right\},\left\{a_{2 m+1}\right\}, \ldots\left\{a_{n}\right\}\right\},\left\{a_{1}, \ldots, a_{n}\right\}=\{1, \ldots, n\}\right\} .
$$

Then by differentiating iteratively we see that

$$
R_{n}=\sum_{m=0}^{\lfloor n / 2\rfloor} \sum_{P \in E_{m, n}} D_{a_{1}, a_{2}} \cdots D_{a_{2 m-1}, a_{2 m}} D_{a_{2 m+1}} \cdots D_{a_{n}} \mathrm{e}^{S_{p}}
$$

Indeed, the formula is clearly true for $n=1$. Moreover, using the fact that, for $1 \leq a_{i}, a_{i+1} \leq n$,

$$
\frac{\partial}{\partial x_{n+1}} D_{a_{i}, a_{i+1}}=0
$$

we have, for each $P=\left\{\left\{a_{1}, a_{2}\right\}, \ldots,\left\{a_{2 m-1}, a_{2 m}\right\},\left\{a_{2 m+1}\right\}, \ldots,\left\{a_{n}\right\}\right\}$ in $E_{m, n}$,

$$
\begin{gathered}
\frac{\partial}{\partial x_{n+1}} D_{a_{1}, a_{2}} \cdots D_{a_{2 m-1}, a_{2 m}} D_{a_{2 m+1}} \cdots D_{a_{n}} \mathrm{e}^{S_{p}} \\
=\sum_{k=2 m+1}^{p}\left(D_{a_{1}, a_{2}} \cdots D_{a_{2 m-1}, a_{2 m}} D_{a_{2 m+1}} \cdots D_{a_{k-1}} D_{a_{k}, n+1} D_{a_{k+1}} \cdots D_{a_{n}}\right. \\
\left.\quad+D_{a_{1}, a_{2}} \cdots D_{a_{2 m-1}, a_{2 m}} D_{a_{2 m+1}} \cdots D_{a_{n}} D_{n+1}\right) \mathrm{e}^{S_{p}}
\end{gathered}
$$

We therefore obtain (8) after summing over $P$ and $m$.

Finally, by taking $n=p$ we have

$$
\frac{\partial^{p}}{\partial x_{1} \cdots \partial x_{p}} \mathrm{e}^{S p}=\sum_{m=0}^{\lfloor p / 2\rfloor} \sum_{P \in E_{m, p}} D_{a_{1}, a_{2}} \cdots D_{a_{2 m-1}, a_{2 m}} D_{a_{2 m+1}} \cdots D_{a_{p}} \mathrm{e}^{S_{p}} .
$$

We now evaluate this expression for $x_{1}=\cdots=x_{p}=0$. Since

$$
\begin{gathered}
D_{a_{i}}(0, \ldots, 0)=\sum_{b=1}^{p} \gamma_{l}^{\omega \varepsilon}\left(u_{b}-u_{a_{i}}\right), \\
D_{a_{i}, a_{i+1}}(0, \ldots, 0)=\gamma_{l}^{\varepsilon}\left(u_{a_{i}}-u_{a_{i+1}}\right),
\end{gathered}
$$


and

$$
\mathrm{e}^{S_{p}(0, \ldots, 0)}=\exp \left(\sum_{1 \leq i<j \leq p} \gamma_{l}^{\omega}\left(u_{i}-u_{j}\right)\right)
$$

we can express the moment $\mathrm{E}\left[X_{l}(t)^{p}\right]$ as

$$
\sum_{m=0}^{\lfloor p / 2\rfloor} \sum_{P \in E_{m, p}} \sum_{b_{1}=1}^{p} \cdots \sum_{b_{p-2 m}=1}^{p} \int_{0}^{t} \cdots \int_{0}^{t} \mathrm{~d} u_{1} \cdots \mathrm{d} u_{p} f_{l}^{m, P, b_{1}, \ldots, b_{p-2 m}}\left(u_{1}, \ldots, u_{p}\right)
$$

where

$$
\begin{aligned}
& f_{l}^{m, P, b_{1}, \ldots, b_{p-2 m}}\left(u_{1}, \ldots, u_{p}\right)=\gamma_{l}^{\varepsilon}\left(u_{a_{1}}-u_{a_{2}}\right) \ldots \gamma_{l}^{\varepsilon}\left(u_{a_{2 m-1}}-u_{a_{2 m}}\right) \gamma_{l}^{\omega \varepsilon}\left(u_{b_{1}}-u_{a_{2 m+1}}\right) \cdots \\
& \times \gamma_{l}^{\omega \varepsilon}\left(u_{b_{p-2 m}}-u_{a_{p}}\right) \exp \left(\sum_{1 \leq i<j \leq p}^{p} \gamma_{l}^{\omega}\left(u_{i}-u_{j}\right)\right) \text {. }
\end{aligned}
$$

Define

$$
\Gamma_{p}(m)=\sigma^{p} \lambda^{p-2 m} T^{\lambda^{2} p(p-1) / 2}\left(c^{\varepsilon}\right)^{m}\left(c^{\omega \varepsilon}\right)^{p-2 m} .
$$

Then, from Property 4, each

$$
f_{l}^{m, P, b_{1}, \ldots, b_{p-2 m}} \uparrow f^{m, P, b_{1}, \ldots, b_{p-2 m}} \quad \text { as } l \rightarrow 0,
$$

where $f^{m, P, b_{1}, \ldots, b_{p-2 m}}$ is given by

$$
\begin{aligned}
& f^{m, P, b_{1}, \ldots, b_{p-2 m}}\left(u_{1}, \ldots, u_{p}\right) \\
&=\Gamma_{p}(m)\left|u_{a_{1}}-u_{a_{2}}\right|^{-2+2 H} \cdots\left|u_{a_{2 m-1}}-u_{a_{2 m}}\right|^{-2+2 H}\left(u_{b_{1}}-u_{a_{2 m+1}}\right)_{+}^{-1+H} \ldots \\
& \times\left(u_{b_{p-2 m}}-u_{a_{p}}\right)_{+}^{-1+H} \prod_{1 \leq i<j \leq p}\left|u_{i}-u_{j}\right|^{-\lambda^{2}},
\end{aligned}
$$

which is integrable if and only if

$$
-1+p H-\frac{p(p-1)}{2} \lambda^{2}>0
$$

Applying the monotone convergence theorem gives the result, with

$$
\begin{aligned}
K(p)= & \sum_{m=0}^{\lfloor p / 2\rfloor} \Gamma_{m}(p) \sum_{P \in E_{m, p}} \sum_{b_{1}=1}^{p} \cdots \sum_{b_{p-2 m}=1}^{p} \int_{0}^{1} \cdots \int_{0}^{1} \mathrm{~d} u_{1} \cdots \mathrm{d} u_{p} \\
& \times\left|u_{a_{1}}-u_{a_{2}}\right|^{-2+2 H} \cdots\left|u_{a_{2 m-1}}-u_{a_{2 m}}\right|^{-2+2 H} \\
& \times\left(u_{b_{1}}-u_{a_{2 m+1}}\right)_{+}^{-1+H} \cdots\left(u_{b_{p-2 m}}-u_{a_{p}}\right)_{+}^{-1+H} \prod_{1 \leq i<j \leq p}\left|u_{i}-u_{j}\right|^{-\lambda^{2}} .
\end{aligned}
$$




\section{Appendix C. Proof of Theorem 4}

\section{C.1. Behavior of $\rho^{(1)}$}

Here and in the following, we will use the identity

$$
\begin{aligned}
\varepsilon_{l}\left(u_{1}\right) & \cdots \varepsilon_{l}\left(u_{p}\right) \exp \left(\omega_{l}\left(u_{1}\right)+\cdots+\omega_{l}\left(u_{p}\right)\right) \\
& =\left.\frac{\partial^{p}}{\partial x_{1} \cdots \partial x_{p}}\right|_{x_{1}=\cdots=x_{p}=0} \exp \left(\omega_{l}\left(u_{1}\right)+\cdots+\omega_{l}\left(u_{p}\right)+x_{1} \varepsilon_{l}\left(u_{1}\right)+\cdots+x_{p} \varepsilon_{l}\left(u_{p}\right)\right) .
\end{aligned}
$$

Applying this identity for $p=2$, Property 4 , and the monotone convergence theorem, yields

$$
\mathrm{E}\left[\delta_{\tau} X(0) \delta_{\tau} X(k \tau)\right]=c^{\varepsilon} \sigma^{2} T^{\lambda^{2}} \int_{0}^{\tau} \mathrm{d} u_{1} \int_{k \tau}^{(k+1) \tau} \mathrm{d} u_{2}\left|u_{2}-u_{1}\right|^{-1+d} .
$$

Note that from the value of $c^{\varepsilon}$ given in Subsection 3.3, $c^{\varepsilon}$ does depend on $d$, but is approximately $\frac{1}{2}$ for small $d$. It is easy enough to compute the integral above, which gives, for $k=0$,

$$
\mathrm{E}\left[\delta_{\tau} X(0)^{2}\right]=\frac{2 c^{\varepsilon} \sigma^{2} T^{\lambda^{2}}}{d(1+d)} \tau^{1+d}
$$

and, for $|k|>0$,

$$
\mathrm{E}\left[\delta_{\tau} X(0) \delta_{\tau} X(k \tau)\right]=\frac{c^{\varepsilon} \sigma^{2} T^{\lambda^{2}}}{d(1+d)}\left(|k+1|^{1+d}+|k-1|^{1+d}-2|k|^{1+d}\right) \tau^{1+d} .
$$

It follows that, for $|k|>0$, the correlation $\rho_{d}^{(1)}(\tau, k)$ is of order $d$ when $d$ is small. More precisely, for $|k|=1$,

$$
\rho_{d}^{(1)}(\tau, k) \sim d \log (2) \quad \text { as } d \rightarrow 0
$$

and, for $|k|>1$,

$$
\rho_{d}^{(1)}(\tau, k) \sim \frac{1}{2} d\left(|k| \log \left(1-\frac{1}{k^{2}}\right)+\log \left(1+\frac{2}{|k|-1}\right)\right) \quad \text { as } d \rightarrow 0 .
$$

\section{C.2. Behaviors of $\rho^{(2)}$ and $\rho^{(3)}$}

From Proposition 4 , it is enough to prove the result for $\rho^{(2)}$. Following the same line as above, we obtain

$$
\begin{aligned}
& \mathrm{E}\left[\delta_{\tau} X(0) \delta_{\tau} X(k \tau)^{2}\right] \\
& =c^{\varepsilon} c^{\omega \varepsilon} \sigma^{3} \lambda T^{3 \lambda^{2}} \tau^{\left(3-3 \lambda^{2}+3 d\right) / 2} \\
& \quad \times \sum_{i_{1}, i_{2}, i_{3}} \int_{0}^{1} \mathrm{~d} u_{1} \int_{k}^{k+1} \mathrm{~d} u_{2} \int_{k}^{k+1} \mathrm{~d} u_{3}\left|u_{i_{1}}-u_{i_{2}}\right|^{-1+d}\left(u_{i_{2}}-u_{i_{3}}\right)_{+}^{\left(-1+d+\lambda^{2}\right) / 2} \\
& \times\left|u_{i_{3}}-u_{i_{1}}\right|^{-\lambda^{2}},
\end{aligned}
$$

the sum being taken over all permutations $i_{1}, i_{2}$, and $i_{3}$ of the set $\{1,2,3\}$. Note that, depending on the sign of $k$ and the permutation, it may be the case that $u_{i_{2}}$ lies in an interval lower than $u_{i_{3}}$, so the corresponding integral is 0 . Also, note that, from the values of $c^{\varepsilon}$ and $c^{\omega \varepsilon}$ given in Subsection 3.3, the product $c^{\varepsilon} c^{\omega \varepsilon}$ is approximately 0.55 for small $d$. 
Taking into account the range of possible values for $d>0$ and $\lambda^{2}$, the integrals above are clearly finite. Moreover, as $d$ goes to 0 , only the integral

$$
I_{d}(k)=\int_{0}^{1} \mathrm{~d} u_{1} \int_{k}^{k+1} \mathrm{~d} u_{2} \int_{k}^{k+1} \mathrm{~d} u_{3}\left|u_{2}-u_{3}\right|^{-1+d}\left(u_{3}-u_{1}\right)_{+}^{\left(-1+d+\lambda^{2}\right) / 2}\left|u_{1}-u_{2}\right|^{-\lambda^{2}}
$$

(and the one where we permute $u_{2}$ and $u_{3}$, which is obviously much the same) explodes for $k \geq 0$, while in the case $k<0, I_{d}(k)$ is exactly 0 , so the moment $\mathrm{E}\left[\delta_{\tau} X(0) \delta_{\tau} X(k \tau)^{2}\right]$ remains bounded. For $k \geq 2$, we have the bounds

$$
\begin{aligned}
(k+1)^{\left(-1+d-\lambda^{2}\right) / 2} \int_{0}^{1} \mathrm{~d} u \int_{0}^{1} \mathrm{~d} v|u-v|^{-1+d} \\
\quad \leq I_{d}(k) \\
\quad \leq(k+1)^{\left(-1+d-\lambda^{2}\right) / 2} \int_{0}^{1} \mathrm{~d} u \int_{0}^{1} \mathrm{~d} v|u-v|^{-1+d},
\end{aligned}
$$

from which we obtain

$$
I_{d}(k) \sim c(k) d^{-1} \quad \text { as } d \rightarrow 0,
$$

where the $c(k)$ are some positive constants such that

$$
2(k+1)^{\left(-1-\lambda^{2}\right) / 2} \leq c(k) \leq 2(k-1)^{\left(-1-\lambda^{2}\right) / 2} .
$$

For $k=0,1$, it can be similarly shown that

$$
I_{d}(k) \sim c(k) d^{-1} \quad \text { as } d \rightarrow 0,
$$

where $c(0)$ and $c(1)$ are some positive constants. From this we can write, for $k<0$,

$$
\left|\rho_{d}^{(2)}(\tau, k)\right|=O\left(d^{3 / 2}\right) \quad \text { as } d \rightarrow 0
$$

and, for $k \geq 0$,

$$
\rho_{d}^{(2)}(\tau, k) \sim-\left(\frac{c^{\varepsilon} T^{3 \lambda^{2}}}{2}\right)^{1 / 2} c^{\omega \varepsilon} \lambda c(k) d^{1 / 2} \quad \text { as } d \rightarrow 0 .
$$

\section{Acknowledgements}

This research was part of the Chair 'Financial Risks' of the Risk Foundation, the Chair 'Derivatives of the Future' sponsored by the Fédération Bancaire Française, and the Chair 'Finance and Sustainable Development' sponsored by EDF and Calyon.

\section{References}

[1] Abry, P., Chainais, P., Coutin, L. And Pipiras, V. (2009). Multifractal random walks as fractional Wiener integrals. IEEE Trans. Inf. Theory 55, 3825-3846.

[2] BaCry, E. ANd Muzy, J. F. (2003). Log-infinitely divisible multifractal process. Commun. Math. Phys. 236, 449-475.

[3] Bacry, E., KozhemyaK, A. And Muzy, J. F. (2012). Log-normal continuous cascades: aggregation properties and estimation. Quant. Finance, 24pp. (electronic).

[4] Barral, J. and Mandelbrot, B. B. (2002). Multifractal products of cylindrical pulses. Prob. Theory Relat. Fields 124, 409-430. 
[5] Bouchaud, J.-P. And Potters, M. (2003). Theory of Financial Risks and Derivative Pricing. Cambridge University Press.

[6] Bouchaud, J.-P., Matacz, A. And Potters, M. (2001). Leverage effect in financial markets: the retarded volatility model. Phys. Rev. Lett. 87, 228701, 4pp.

[7] Calvet, L. E. And Fisher, A. J. (2008). Multifractal Volatility: Theory, Forecasting, and Pricing. Academic Press.

[8] Chan, G. And Wood, A. T. A. (1998). Simulation of stationary Gaussian vector fields. Statist. Comput. 9, 265-268.

[9] Ciliberti, S., Bouchaud, J.-P. and Potters, M. (2008). Smile dynamics - a theory of the implied leverage effect. Preprint. Available at http://arxiv.org/abs/0809.3375v1.

[10] Cont, R. (2001). Empirical properties of asset returns: stylized facts and statistical issues. Quant. Finance 1, 223-236.

[11] Duchon, J., Robert, R. And Vargas, V. (2010). Forecasting volatility with the multifractal random walk model. Math. Finance 22, 83-108.

[12] Frisch, U. (1995). Turbulence. Cambridge University Press.

[13] Frisch, U. ANd PARISI, G. (1985). Fully developed turbulence and intermittency. In Proc. Internat. Summer School Phys. Enrico Fermi, North-Holland, Amsterdam, pp. 84-88.

[14] Ludeña, C. (2008). $L^{p}$-variations for multifractal fractional random walks. Ann. Appl. Prob. 18, $1138-1163$.

[15] Mandelbrot, B. B. (1997). Fractals and Scaling in Finance. Springer, New York.

[16] Masoliver, J. ANd Perelló, J. (2002). A correlated stochastic volatility model measuring leverage and other stylized facts. Internat. J. Theoret. Appl. Finance 5, 541-562.

[17] Muzy, J. F., Delour, J. ANd Bacry, E. (2000). Modelling fluctuations of financial time series: from cascade process to stochastic volatiliy model. Europ. Phys. J. 17, 537-548..

[18] Perelló, J., Masoliver, J. And Bouchaud, J.-P. (2010). Multiple time scales in volatility and leverage correlations: a stochastic volatility model. Appl. Math. Finance 11, 27-50.

[19] Pochart, B. And Bouchaud, J.-P. (2002). The skewed multifractal random walk with applications to option smiles. Quant. Finance 2, 303-314.

[20] Robert, R. and Vargas, V. (2008). Hydrodynamic turbulence and intermittent random fields. Commun. Math. Phys. 284, 649-673. 